\title{
"Such Conversations Are Not Done in the Families": A Qualitative Study of the Determinants of Early Adolescents' Access to Sexual and Reproductive Health and Rights Information in Rwanda.
}

Valens Mbarushimana ( $\nabla$ mbavalens@gmail.com )

School of Public Health, Faculty of Health Sciences, University of the Witwatersrand, Johannesburg Daphney Nozizwe Conco

School of Public Health, Faculty of Health Sciences, University of the Witwatersrand, Johannesburg Susan Goldstein

SAMRC/Centre for Health Economics and Decision Science, PRICELESS SA, School of Public Health, Faculty of Health Sciences, University of the Witwatersrand, Johannesburg

\section{Research Article}

Keywords: gender, sexuality, education, early adolescent, sexual and reproductive health and rights, Rwanda

Posted Date: November 17th, 2021

DOI: https://doi.org/10.21203/rs.3.rs-1043620/v1

License: (c) (i) This work is licensed under a Creative Commons Attribution 4.0 International License. Read Full License 


\section{Abstract \\ Background}

Acces to sexual and reproductive health and rights information during adolescence is a basic human right. This study investigated the key informants' perspectives on the benefits, sources of information, and the determinants (enablers and hindrances) of sexual and reproductive health and rights information among early adolescents in Rwanda.

\section{Methods}

a qualitative study using semi-structured interviews with purposively selected public and private key informants in Rwanda was conducted. Interview transcripts were imported in Nvivo 11 for thematic analysis.

\section{Results}

In total, sixteen interviews involving an equal number of male and female participants were conducted. Despite it being perceived as a taboo topic, participants acknowledged sexuality education as beneficial for early adolescents and confirmed that there are various channels for accessing gender and sexuality information. The school comprehensive sexuality education was depicted as a common source of information, while parents were not. Participants highlighted disparities in information access between rural and urban settings. Faith-based organizations and public institutions did not share the same perspective on the topics that should be covered in gender and sexuality education. Faith-Based Organizations focused on moral values of abstinence and virginity, while public institutions emphasized ways of having safe sex including the use of modern contraceptive methods. Participants noted the need to address the confusion about the concept of gender. Participants' reflections on gender dynamics revealed that compared to girls, boys culturally override sexual health. Participants thought that access to gender and sexuality information by early adolescents depended on multiple factors at the individual (curiosity, age, behaviours), relationships (discussions with parents, peer sexual norms), community (the content of the curriculum, culture, religious beliefs), and societal (economic challenges, policy, and laws) levels.

\section{Conclusion}

Participants from various backgrounds acknowledged the benefits of sexuality education, as well as the various channels available to younger adolescents for accessing SRHR information, even though their perspectives on the content differed. The study's multi-layered analysis revealed potential areas for improving early adolescents' access to gender and SRHR information. 


\section{Background}

Access to sexual and reproductive health and rights (SRHR) education during early adolescence is a basic human right. The International Conference on Population and Development (ICPD) held in Cairo in 1994 called for comprehensive sexuality education (CSE) of adolescents to respond to their need for information and skills for a better adolescent sexual and reproductive health and rights [1]. This education aims to deliver information on human sexuality, sexual and reproductive health (SRH), gender equity, and human rights; and to help adolescents to adopt safe and responsible sexual behaviours [1]. Timely access to high-quality SRHR information plays a central role in healthy behaviours and safer sexual practices among adolescents [2]. In this regard, early adolescents need accurate information about biological, sociocultural, psychological, relational, and spiritual dimensions of sexuality [3].

Gender and sexuality discussions intensify during early adolescence providing a window of opportunity in terms of gender equality before these roles and related behaviours become well-established $[4,5]$. Gender and sexual behaviour are closely interconnected. Gender is a determinant of the sexual behaviours of adolescents [6-9]. Gender embodies social norms, attitudes, and activities that society deems appropriate for boys and girls [10]. Family, media, and social institutions teach boys and girls appropriate behaviours at an early age $[10,11]$. Sexual behaviour is shaped by cultural discourses and socially constructed attitudes and ideas around it that change over time [10,12]. Gender inequalities and social norms limit young adolescents' sexual decision-making power and have been associated with negative SRH outcomes (pregnancy, STIs) among adolescent girls [7, 9, 11]. In addition, while gender socialization starts earlier in childhood, attitudes towards gender roles intensify during early adolescence $[4,13]$.

The gender and sexuality socialization process takes place in the socio-ecological environment of early adolescents [14]. It is therefore important to consider this large environment of adolescent health to understand the determinants of early adolescents' access to gender and sexuality education in Rwanda. This environment influences early adolescents' sexual health from several layers including the individual, relationships, community, and society levels [15].

In Rwanda, the number of information channels for early adolescents and the general population has significantly increased over the last decades. Significant progress has been achieved in media and information and communication technology, with an increase in licensed radio broadcasters, TV stations, internet subscriptions, and mobile telephony penetration [16]. However, the role of such media in delivering SRHR education to early adolescents is not well known. Furthermore, Rwanda has defined different policies to promote child and adolescent health [17-19]. Primary education is mandatory and free in public schools [20] and comprehensive sexuality education has been delivered in primary and secondary schools since $2016[18,21]$. The purpose of CSE is to increase knowledge of sexuality, gender, and other reproductive issues including sexually transmitted infections [22]. However, there is limited evidence on the determinants of access to SRHR information among early adolescents. 
Globally, adolescents face challenges including limited access to sexual and reproductive health (SRH) information resulting in poor SRH knowledge, early marriage, pregnancy, poor decision-making in SRH matters, and poor quality of SRH services [23]. In Rwanda, five per cent of female adolescents and $11 \%$ of male adolescents engage in sexual activity before they are 15 years old [24]. Pregnancy among schooling girls (10-18 years) remains a concern and is reported countrywide [17]. Young people (14-24 years) lack adequate SRHR knowledge, experience transactional sex, and believe that obtaining SRH services is socially prohibited [25].

Previous studies on early adolescents have focused on gender norms [26-28] and attitudes [4]. Such studies have not focused on early adolescents' access to SRHR information in the broad context of their health. The purpose of this study was to explore stakeholders' views on the benefits, sources of information, and the determinants (enablers and hindrances) of sexual and reproductive health and rights information among early adolescents in Rwanda.

\section{Methods}

\section{Study setting}

This study was conducted in Rwanda. Rwanda is a landlocked country located in Eastern Africa, with a surface area of 26,338 square kilometres. Rwanda is bordered by Uganda in the North, Tanzania in the East, Burundi in the South, and the Democratic Republic of Congo in the West [29]. The Fifth Rwanda Population and Housing Census of 2016 indicated that $52 \%$ of the population is younger than 20 years, with early adolescents (10-14 years) consisting of $13.7 \%$ of the general population [30]. The Rwandan population is mainly rural (83\%) [31]. The age of sexual consent and legal marriage is respectively 18 and 21 years [32].

\section{Study design}

This study used qualitative research methods, semi-structured interviews with key informants to explore stakeholders' views on educating early adolescents on SRHR, the sources of SRHR information, determinants of and barriers to early adolescents' SRHR in Rwanda.

\section{Sampling and recruitment of participants}

A purposive sampling strategy was adopted to recruit participants whose positions and/ or expertise were involved, among other things, in disseminating gender and sexuality information to early adolescents and youths in Rwanda. The eligible participants were selected from public institutions at central and local levels, Faith-Based Organisations (FBO) and non-governmental organizations (NGO's) in Rwanda. 
The public institutions included: Ministry of Education; Ministry of Gender and Family Promotion; Ministry of Sports and Culture; Ministry of Health; Rwanda Biomedical Centre (RBC); Rwanda National Children's Commission; Rwanda Education Board (REB); and Districts of Gasabo and Nyarugenge, the FBOs including the Roman Catholic Church, the Seventh-Day Adventist Church, the Pentecostal Church (ADEPR), and the Rwanda Muslim Community, and one non-government organization (NGO) was represented in the final study sample.

\section{Data collection}

Data collection was conducted between July and October 2019. Before the interview, requests for conducting interviews were officially sent to participants' institutions. After approval by these institutions, the researchers undertook necessary arrangements to contact participants and determine the modalities of interviews. Before the interview, participants were given the study information and time to ask relevant questions. Each participant was invited to consent to the interview by signing a written consent form. With participants' approval, interviews were digitally recorded. Otherwise, the interviewer took written notes of the interview. The language of the interview depended on the participants' choice. Fifteen interviews were conducted in Kinyarwanda and one interview was conducted in English. Each interview lasted between 30 and 60 minutes.

The interview guide covered several topics including participants' views about the benefits or harms of delivering SRHR information to early adolescents; what they considered the sources of and barriers to SRHR information; their understanding of the determinants of access to SRHR information for this agegroup population.

\section{Data management and analysis}

Audio-recorded interviews were transcribed verbatim in Kinyarwanda. The transcripts were translated by, professional translators into English. Translated interviews were imported in QSR Nvivo 11 for Windows and analyzed inductively and deductively. The researchers read interview transcripts several times to familiarize themselves with the content of the interviews. Next, the researchers started creating and condensing meaning units that were later organized into codes. These codes were then organized into sub-themes, which finally were gathered to generate themes [33, 34].

\section{Results}

\section{Study participants}

The sample included a wide range of participants from public institutions, faith-based organizations, and non-governmental organizations (NGOs) operating in the field of adolescent sexual and reproductive health and rights. In total, sixteen key informants participated in the study. There were nine participants (6 
females and 3 males) from public institutions, six participants from FBOs (five males and one female) and one female participant from NGO.

\section{Perceived determinants of early adolescents' access to SRHR information}

\section{Individual factors}

\section{Need for information}

Participants discussed several factors related to early adolescents' access to SRHR information at the individual level. Need for SRHR information, the participants reported that early adolescents are curious and seek information from various sources including their parents. Nevertheless, the study noted that early adolescents are often not able to satisfy their curiosity through their parents and thus harbour misconceptions. Early adolescents feel shy to ask questions and think their parents would consider them disrespectful. In addition, participants felt that early adolescents do not know where to obtain the right information about gender and sexuality despite their curiosity about SRHR.

"They [early adolescents] do not know that they need information. When we talk to them at school, we find out that they are curious, but give us some wrong information about sexual and reproductive health. They do not know that they need the right information". (P16, Male, Public Institution).

"They are not comfortable discussing their reproductive issues. They believe that parents would consider them as disrespectful, bad-mannered people, uneducated". (P10, Female, Public Institution).

\section{Age of initiating SRHR education}

Participants indicated that the age of initiating gender and sexuality education is crucial. Nevertheless, they did not agree about the age. Some participants felt that such education should be delivered at a young age without indicating the exact time and according to children's age. While other participants felt that ten years should be the age of initiating SRHR education.

"Even before they grow in early adolescents, they should be taught about gender and sexuality according to their developmental age". (P01, Female, Public Institution)

"We realized that at 10 years, children are curious and start being exposed to those challenges of sexual and reproductive health information. Parents can prepare their children by starting to talk to them about sexuality." (P16, Male, Public Institution).

\section{Behaviours}


Finally, the participants felt that early adolescents' behaviours (like kissing and hugging) may have implications for their sexual health, including early pregnancy. Furthermore, some participants from FBOs and public institutions perceived that early adolescents learn some behaviours from music celebrities and renowned journalists who serve as their role models. Learned behaviours sometimes inspire early adolescents' identity, behaviours and sexual experience.

"We teach them but we cannot say that we have reached a point where our children do not do those bad things. They tend to hug and kiss each other, which might lead to more actions. Some of them get pregnant earlier in primary, or secondary school, even in church choirs." (P11, Male, FBO)

"They [Early adolescents] search for information about football or movies celebrities to know them more and choose those who can be their role models". (P01, Female, Public Institution)

\section{Relationship factors}

\section{Parent-child interaction}

The findings indicated that sexuality is not a topic for discussion with children at the family level. The participants indicated that parents instead refer girls to other family members, such as aunties for specific sexuality information. In this perspective, aunties focus on sexual and reproductive health, norms, preparation for marriage, and good motherhood. On the other hand, boys have no specific sources for such information and figure out their sources of information. Furthermore, recognizing that parents' knowledge and attitudes play a key role in delivering gender and sexuality education to early adolescents, participants noted that some parents lack adequate knowledge to deliver gender and sexuality information to early adolescents. Participants felt that educated parents are more likely to provide SRHR information.

"Parents do not have enough information. It might be that we are asking parents to teach children while they don't have that knowledge as well." (P14, Female, Public Institution).

Furthermore, participants noted that parental reluctance to engage with their children is due to the belief that their children may start behaving badly once informed. According to the participants, such belief is rooted in the cultural beliefs that gender and sexuality education induces children in sexual activity.

"People think that giving information to children means encouraging them to have sex". (P16, Male, Public Institution)

Changes in family living conditions have resulted in absence of parents from their homes and the use of housekeepers who cannot adequately address children's education needs. All participants noted that parents lack adequate time to care for their children. According to them, parents can introduce sexuality topics when spending more time with their children. Furthermore, participants highlighted that parents 
may be negative role models, who keep blaming early adolescents for their mistakes rather than preaching by good examples of their actions.

"I have noticed that different topics can come up when parents talk to children for a long time. However, when time is short, they do not. Parents reach home tired, asking if children did their homework and if they had appropriate meals. That is it". (P08, Male, FBO)

"There are some parents, teachers, relatives of those adolescents who teach them what they do not do and who keep giving advice that does not turn into actions." (P01, Female, Public Institution).

Language is a concern in that there is a lack of appropriate language about genital parts in Kinyarwanda. The participants noted that words on this topic are very rude, uncommon, and hard to say to children. They indicated that they use alternative words in Kinyarwanda. Nevertheless, the participants said they were comfortable talking about sexuality in foreign languages such as French or English. Findings further suggested that challenges related to the language can be addressed by using a guide entitled 'Tuganire Mwana Wanjye' [Let's Talk My Child]. The purpose of the guideisto help parents to find the appropriate word to facilitate parent-child discussion on sexuality education in the local language, Kinyarwanda.

"For instance, saying the word 'penis' in Kinyarwanda is considered a problem; but it sounds fine in French or English". (P16, Male, Public Institution).

In addition, the findings revealed that it is culturally inappropriate that parents to deliver gender and sexuality information to children. Accordingly, sexuality is a taboo topic and has been that way from generation to generation.

The Rwandan culture influences parents to not talk to their children about reproductive health. Many people don't talk about it. It is a taboo subject. It is due to the way people are raised." (P10, Female, Public Institution).

\section{Peer norms}

The findings indicated that early adolescents receive some normative information from their peers which has implications on their sexual health outcomes. One of these norms is the general expectation that girls should not talk about having sex, leaving the decision-making power to boys. Accordingly, girls remain silent about their sexual needs. The participants noted that this silence compromises girls' rights, especially those related to access to information on safe sexual relationships with peers. The study also highlighted the existence of other harmful sexual norms. While boys perceive girls as money eaters, girls believe that boys are providers and hunters for sex. According to participants, the consequence of these norms is that girls need to have sex if they get any money/presents from boys.

"They [boys and girls] never set a talk on whether the boy wants to have sex. In some cases, the girl also wants it and it is her right to say she wants it. The problem is that general expectation that a girl should never talk about having sex". (P02, Female, NGO) 
"Girls grow up shy because culture expects them not to speak in public. Therefore, it is easy for anyone to deviate or fool them because they cannot speak up as the society expects them to always be quiet". (P14, Female, Public Institution)

"Boys should always pay everything for the girl. This is associated with the idea that if he spends money on a girl according to the norm, she should also pay him back. Therefore, he expects that pay like to have sex." (P02, Female, NGO)

Finally, there are peer pressures that contradict the social expectation of remaining virgin for girls until marriage, sticking to one girlfriend, proving love through sexual intercourse, and keeping sexual relationships with boy/girlfriends.

"Peers spread messages that are in contrast with the social culture or expectation of being virgin until marriage. For example, a real boy has sex with multiple girls; or similarly for a girl: you're not a real girl until you lose your virginity". (P13, Female, Public Institution)

\section{Community-level factors}

\section{Content of the curriculum}

Although most participants delivered SRHR information to early adolescents, there was disagreement about the content of the information. While churches focus on the 'Word of God' that prohibits premarital sexual activity, participants from public institutions suggested that churches should go further and teach early adolescents about having safe sex. They indicated that SRHR education should also encompass modern contraceptive methods in addition to the natural ones promoted by FBOs. The participants from FBOs focus on the moral values of virginity and chastity while government education introduces condom use.

"The church education focuses on moral values. We teach them by good examples of their grandparents, parents, elder brothers, and sisters who resisted rape until they died for it; they refused to break their virginity. We teach them about virginity and chastity." (P07, Male, FBO).

"There are people who only teach that having sex is a sin without explaining more. They do not go beyond that to suggest means for safe sex."(P16, Male Public Institution)

"We need to avail emergency contraceptives to early adolescents who fail to abstain from sexual intercourse to prevent unplanned pregnancy. (P16, Male, Public Institution)

\section{Culture}

The findings noted the patriarchial culture, where children belong to their fathers, imposes differences in gender roles and responsibilities between girls and boys. Such disparities favour boys more than girls. The study indicated girls are supposed to perform mainly home activities while boys are assigned 
manly tasks that generate income. Despite equality of men and women in law, some participants indicated that gender equality is not achievable given their differences in nature and cultural expectations. The study also highlighted that both boys and girls face a different kind of pressure related to social expectations, mainly those related to marriage.

"Men and women cannot be equal by nature, image and powers. I do not deny their equality by law. However, family responsibilities make them unequal". (P09, Male, FBO)

"For example ..., boys have to handle all problems. They have to work hard and build a house, then get married. Girls face a cultural challenge of early marriage. It is believed that when girls do not get married earlier they will not survive. They marry husbands they have never thought of." (P08, Male, FBO)

"You find a boy is given freedom, while the girl is denied freedom. To boys having sex is not a big concern, which is different for girls. However, it is a concern to both of them as they are all affected by having sex". (P16, Male, Public Institution).

The findings also indicated that the culture contributes to uneven gender considerations in terms of decision-making power. The participants depicted men or boys as owners of power and decisions to initiate sexual relationships while women or girls were subject to submission. Accordingly,boys tend to appear as protectors of girls while the latter act as weak even when they are strong, avoiding boy-like behaviours.

"Culturally... it is well known that husbands are strong to protect their wives. Even if a wife is strong, she has to act simply and flexibly. She expects and enjoys protection from her husband (P01, Female, Public Institution)

In addition, the study noted that compared to girls, boys enjoy more power over their sexual health. However, participants recognized that women have the right to make sexual decisions to avoid related health risks. Given this unequal decision-making power, boys might abuse these powers through initiating early sexual intercourse. The study also indicated that it is taboo for women and girls to express their sexual desires.

"Men are entitled to making decisions about their sexual health while women are not. ... from culture to reality, everyone who has a normally functioning brain has the right to take decisions mainly those healthrelated ones." (P01, Female, Public Institution)

"Boys are expected to initiate of relationships with girls. They may fall into early sexual intercourse with young girls because of the perception that being a man is showing their strength to girls". (P05, Male, Public Institution).

"It is taken as a taboo [for a woman] to tell her husband that she wants sexual intercourse while as a human being, she might also wish to have sex". (P01, Female, Public Institution). 
Finally, the culture maintains some sayings that nourish imbalances in sexual decision-making powers. One of these sayings is about the irresistibility of men towards their sexual drive and their entitlement to multiple sexual partners. Such a saying has some implications for young boys and girls' behaviours who grow in an environment where such beliefs still hold.

"A Rwandan proverb says that bulls are irresistible whenever they want sex (Imfizi ntiyimirwa'). Boys grow up as such and behave like their father with such beliefs." (P01, female, Public Institution).

\section{Religious beliefs}

The findings indicated that religions have a role in delivering gender and sexuality information to early adolescents. The study indicated that some churches deliver gender and sexual and reproductive health information which influences early adolescents' knowledge, beliefs, and behaviours. However, some churches are reluctant to teach about sexuality, regarding it as a sin. According to the findings, FBOs consider that providing sexual and reproductive health education to early adolescents promotes immorality and violates God's commandment of abstaining from sex. Furthermore, FBO participants indicated that delivering such information may encourage early adolescents to have premarital sex.

"The church education focuses on moral values. We teach by good examples of their grandparents, parents, elder brothers, and sisters who resisted rape and refused to break their virginity. We teach early adolescents to abstain from having sex, remain virgin and chaste using examples of the Virgin Mary and other Saints who died because of protecting their virginity." (P07, Male, FBO)

"Teaching children to use a condom means inviting them to have protected sex. If the commandment says not to abstain from sex, it would be a contradiction because whether sex is protected or not, that commandment would be violated. This is immoral, misbehaving". (P11, Male, FBO)

Some participants highlighted that the misunderstanding of the Holy Scriptures contributes to gender inequality. They believe that some church members misinterpret the Holy Bible by saying that men have authority over women and take it as a rule.

"Some of us who are not able to explain well the Bible tells you that a husband has authority over his wife and take this as their guiding principle. Even their wives accept it as such. It is taken as a rule set by God while this is a misinterpretation of the Holy Scriptures." (P07, Male, FBO)

\section{Societal factors}

\section{Economic challenges}

According to participants, economic factors such as financial means were related to early adolescents' access to gender and sexuality information. The study specifically highlighted that inadequate financial resources to generate information tools such as books and training modules were an important challenge to enable early adolescents' access to gender and sexuality information. They also noted that even the 
existing teaching materials were not adequately designed for local realities. They further noted issues of shortage of skilled and qualified personnel, as well as the inability to exploit available communication channels including social media in delivering SRHR information.

"It also requires financial means. Books are expensive... Modules require time to generate." (P17, Female, Public Institution)

"There are no materials unless those found in biology but it's not also from our culture. They are most often brought from different NGOs. The package from NGOs is always wrong. You may find in the preparation a white woman sitting around, and it is a problem." (P08, Male, FBO)

"We have a shortage of employees to deliver sexuality information....Another barrier is technology use. We would like to send that information using WhatsApp and other social media but it is still expensive and children do not access it on phones." (P16, Male, Public Institution).

\section{Policies and laws}

Participants also raised discrepancies in policy-makers views about instilling family values in early adolescents. To illustrate this, one participant gave an example that some policy-makers support women's dressing styles and their rights to dress the way they wish; while some others believed that women's dressing styles sometimes compromise the ideals of the family through exhibiting "their private parts" to other people, especially children. According to that participant, while children need a family that serves them as a model, some policy-makers believed that letting people (especially women) dress the way they want does not serve this purpose. The participant raised the need to address this confusion in family ideals and the respect of rights to dressing styles.

"Even family policy-makers have a divided perspective on the ideal family. One says that parents should be exemplary to children by their dressing styles. Another says that culture evolves; people have the right to dress the way they want and to display their beautiful body parts, including their breasts. Commenting on women who are 'naked' in front of their children means violating their human rights, especially women's rights. There is confusion in our society!" (P05, Male, Public Institution).

Furthermore, the participants criticized the processes of setting gender and sexuality policies as inadequate, as they do not involve people from all layers of the community including the elderly people. The findings indicated the need to set evidence-based policy through research. They noted that some of these policies were imported from the Western World and rapidly implemented in settings where they sometimes have no relevance. Participants also noted that policy content should reflect community members' perspectives.

"The family members, especially elderly people from the villages, are not consulted. If they [policymakers] could approach them, they would learn about what used to happen 20 years ago, how women behaved towards their husbands and how the husbands behaved towards their wives, and what made them keep their family secrets." (P05, Male, Public Institution) 
"Policy content should not be imposed. Policy-makers abruptly come up with content from European countries. They do not research to get informed about the local context to include community members' cultural perspectives." (P08, Male, FBO)

Concerning laws, participants from public institutions noted the need to solve specific reproductive health problems and revise laws to allow children to make use of emergency contraceptives when needed.

"We should also revise current laws to support children who have difficulty to abstain from sex and need emergency contraceptives." (P16, Male, Public Institution).

\section{Participants' views and perceptions on SRHR information}

The participants reflected on the role of gender and sexuality education in moulding early adolescents' behaviours and their future life, stating that knowing that their bodies are changing, give them opportunities to manage those changes through appropriate decision-making. The participants also indicated that SRHR education has the potential to facilitate early adolescents' positive attitudes towards their sexuality, enabling them to better manage their sexual instincts, the relationships between boys and girls, as well as dynamics associated with mutual sexual attraction. The SRHR information was perceived by participants, not only as means to promote the well being of early adolescents, but that of their families and wider society. Participants stated that sexuality education is meant to enable recipients to plan for their health, particularly their sexual and reproductive health. Hence the view that it is crucial for the general wellbeing of the wider society, as it is believed that such education enables people better plan when to have offsprings.

The participants noted the role of gender and sexuality education in creating awareness of similarities and differences between boys and girls, suggesting that such self-awareness increases recipients' ability to exercise their rights in the context of culturally accepted inequalities between boys and girls.

“Gender education increases early adolescents' self-awareness and the knowledge of their rights. Our culture imposes inequality between a boy and a girl. Changing that mindset is a journey to equality".(P15, Male, Public Institution)

"Adolescents face many challenges that they can solve only if they know. They can be aware of the right time to do sex and how to protect themselves against sexually transmitted diseases, as well as other consequences. Teaching them earlier helps them to make informed decisions". (P16, Male, Public Institution)

The participants further noted how gender and sexuality education contribute to increased knowledge of SRHR related needs and rights among adolescents while providing the necessary support.

"During menstruation, some adolescent girls fall sick, and school performance slows down. A society that is aware of this provides special support to that adolescent girl. They know specific needs and challenges 
associated with this developmental stage including sanitary pads"(P03, Female, FBO)

Providing sexuality and gender education was also viewed as an opportunity to start early to prepare adolescents for marriage and family life.Correspondingly gender issues were considered a concern for both boys and girls, hence the proposal to educate adolescents about teamwork and building strong family relations when they get married.

"This education help early adolescents to apply these principles in their daily life as we prepare them for marriage during this period of early adolescence. Gender introduces teamwork and is key in building a strong family when married". (P03, Female, FBO)

The need for gender education specifically to address unequal distribution of tasks between boys and girls was highlighted. Participants felt that it was important to get early adolescents aware that no specific domestic task should be devoted to boys or girls, arguing that gender education is needed to address the issues of boys' sexual harassment and lack of respect towards girls.

"Sometimes boys harass girls because they were not given lessons about respecting their colleagues [girls]. When boys are not timely educated about respecting girls, they may induce them in sexual intercourse, which may result in early pregnancy and school dropout". (P12, Male, FBO)

Concerning the concept of gender, the participants were of the view that this concept remains obscure in the context of Rwanda, due to the lack of its standard definition. They noted that the community does not clearly understand the meaning and dimensions of gender. Participants felt that the concept of gender has several ideological meanings that are not translatable to Kinyarwanda. The concept was therefore found to be difficult to teach to early adolescents who learn better from things that can be verified, rather than ideologies. This lack of a standard definition of gender hampers the selection of gender content to be taught to early adolescents.

"There is still a problem of lack of standard definition of gender. What is the appropriate subject to teach about it? It is still a problem as long as there is no clear definition of gender in our context". (P08, Male, FBO)

\section{Sources of gender and SRHR information}

The participants indicated that early adolescents access SRHR information through multiple sources. The findings indicated that these sources have diversified because of technological advancement. Some of the sources that have emerged include mobile phones and television. These sources inform early adolescents in many ways. However, given the diversification of sources of information on gender and sexuality for early adolescents, especially online sources, participants noted that the Government of Rwanda has established a policy to regulate the use of these online sources by children to prevent access to harmful content. A participant from an FBO indicated these various sources as follows: 
I think they [early adolescents] get such information from their friends, parents. Schools deliver such information as well. They also access information through information and communication technology, the internet, mobile phones, YouTube, and others. (P03, Female, FBO)

There is a policy called 'Child Online Protection Policy' to protect children from using the internet abusively (P01, Female, Public Institution).

Furthermore, the participants noted the schools as the sources of SRHR information through the comprehensive sexuality education (CSE), whereby gender and sexuality are taught as crosscutting courses with a different weight in the curriculum. According to the participants, the school setting also offers an opportunity to early adolescents to learn about gender and sexuality through gender and health clubs. Gender clubs teach about gender issues including gender equality; while health clubs are concerned with many health issues including sexual and reproductive health issues, prevention of HIV/AIDS, hepatitis, and nutrition among others. Nevertheless, the content of the CSE remains a concern for some participants. Participants noted that the selection of CSE requires the respect of culture and inputs from education stakeholders. They indicated that some aspects of the CSE curriculum such as the dating scenarios might have consequences on early adolescents' behaviours. Participants believe that dating activities may result in sexual activities if children are not adequately overseen in both the school and family environments. In addition, participants were concerned that involving students in scenes of dating each other and doing some discoveries is against morals and culture.

"Some lessons may invite students into early sexual activity and/or early pregnancy when it comes to inclass dating exercises and reaching an agreement on having safe sex using a condom. It becomes hard to continue protecting them from sexual activity on their way back to their families or later." (P08, Male, FBO)

Despite reporting that adequate SRHR information should be delivered in schools, the findings indicated that teachers face some challenges including their personal beliefs and attitudes, and lack of skills to deliver such education.

"We know in many schools, this is not implemented the way it should, because personal beliefs of teachers, because of lack of skills". (P02, Female, NGO)

In addition to schools, the participants highlighted health facility-based SRHR information delivery to early adolescents through youth corners:

"We trained at least two people in every health centre. The program is in place. Early adolescents receive information on sexual and reproductive health from the youth corner, at the health facility level." (P16, Male, Public institution).

Radio and television and the use of mobile phones were reported as important means to deliver SRHR information to early adolescents in general. These radio programs include "Urunana" and "Ni Nyampinga". Urunana targets the general population while Ni Nyampinga focuses on delivering SRHR 
information to young girls. Ni Nyampinga focuses on girls' abstinence and/or use of a condom. This program calls young girls to use abstain from sex or use condoms in case abstention fails. Furthermore, "Mobile for Reproductive Health" (m4RH) is another program through which adolescents, including the younger ones, access SRHR. Using mobile phones, m4RH sends SRHR messages to children.

Furthermore, the participants reported that early adolescents seek SRHR information from social media, including YouTube, Facebook, WhatsApp, and the internet. However, they noted that some of these internet sources spread incorrect SRHR information. The participants highlighted disparities in SRHR sources between rural and urban settings. They revealed that early adolescents from the urban setting have social media as their main sources of information while those from rural settings rely on schools as their main sources of gender and sexuality information.

"Concerning reproductive health, they may get wrong information that may lead them to early sexual intercourse. I have heard about two bad women who always teach about the labia elongation culture on the internet". (P03, Female, FBO)

The participants noted the role of various governmental, local, and non-governmental leaders in delivering gender and sexuality information to early adolescents. They however noted the need to harmonize delivered SRHR information and consider their audiences, namely their age.

Furthermore, the participants indicated that the churches delivered SRHR information to early adolescents on a part-time basis and about the "Word of God" and avoiding premarital sex.

Churches organize seminars for young children and early adolescents, but not regularly. As churches focus on the Word of God, they call them not to have illegal sexual activity. (P03, Female, FBO)

\section{Discussion}

This research sought to explore gender and sexuality stakeholders' perspectives on the determinants of early adolescents' access to SRHR information in Rwanda. The findings revealed that access to SRHR information in Rwanda is embedded in a vast environment of interrelated factors aligning in the socioecological framework of adolescent health [15, 35-37].

The study highlighted some factors influencing early adolescents' access to SRHR at the individual level. It was found that despite their curiosity to seek gender and sexuality information, early adolescents' SRH knowledge was perceived as inadequate in many settings such as Sub-Saharan Africa [38, 39], Guangzhou City in China [40], and Korea [41]. The study also highlighted that key family members restrict early adolescents' attitudes towards seeking SRH information. In this regard, previous research noted that adolescents were being reprimanded for talking about sex to their parents [42]. In addition, the findings noted age as a determinant of accessing SRHR information. While such information should be ageappropriate [1], some studies argue early adolescents are too young to receive SRHR information $[43,44]$. 
Furthermore, a study found an association between the younger age of early adolescents and insufficient SRHR knowledge[41].

This study indicated the role of family members and peer norms influence their access to SRHR information. The findings noted that parents have worries that SRH education would stimulate sexual activity among early adolescents. Studies have indicated that parents and other community members are crucial in shaping early adolescents' attitudes and behaviours related to gender norms and values, and sexuality [45]. Previous research indicates that parental attitudes, especially openness towards sex, influence early adolescents sexual behaviours [41]. Evidence suggest also those early adolescents who report having talks about sexuality with their parents have greater knowledge compared to those who do not [41]. Previous research has indicated that such communication between parents and early adolescents takes place less often due to parents' lower level of education [46], embarrassment, lack of parental knowledge, cultural norms, taboos $[23,43,44,47,48]$, believing that early adolescents are too young to understand $[43,49]$, and the perception that communication about sexuality would encourage sexual experimentation [35, 42, 43, 47, 49]. In contrast, evidence suggests that sexuality education for early adolescents impacts positively on safer sexual behaviours; delays sexual initiation, and increases condom use $[45,50]$.

This study also highlighted the parents' absence from homes reduces parental monitoring of children and opportunities to discuss gender and sexuality matters. Studies noted that spending more time with early adolescents reduces peer influences $[41,51]$. The family provides standards of sexual conduct [52] through conveying sexual information, influencing sexual attitudes, values, and beliefs; and instilling a model of communication about sexual issues [53]. This parental influence is possible through spending more time with early adolescents $[44,54]$.

This study indicated the role of the culture in hindering the discussion between parents and children about gender and sexuality, which is common in Sub-Saharan Africa [47]. This results in sending adolescent girls to other extended family members (aunts, uncles, and grandparents) for sexual socialization [11,44,55], while adolescent boys in Rwanda have normally no reference for related information [54]. While studies emphasize the protective role of parental relationships on adolescent health [42], some parents are reluctant to discuss sex and sexuality due to cultural values that sexuality is a taboo subject and assuming girls' sexual innocence and abstinence until marriage [42]. Research indicates that some parents perceive premarital sexual activity as bad, shameful, and morally reprehensible, which explains their unwillingness to discuss sexual health topics with their children [42]. Studies also indicate that parents have challenges related to lacking the correct language to use, worries about saying too much than needed, fears of difficult questions (e.g. how babies are made), discovering unknown behaviours, and feeling embarrassed $[43,49]$. This study noted that to address the language barrier, a booklet in the local language, Kinyarwanda, was available to facilitate discussion between parents and children on SRHR [56]. 
While the constitution of the Republic of Rwanda recognizes the principle of gender equality between men and women [20], this study indicated differences in decision-making power and gender roles of boys and girls. The study indicated that compared to girls, boys exercised more sexual decision-making power than girls. Girls hold an ambivalent or silent attitude about sexual activity. Studies have underscored that girls are socialized to be submissive, and they were unable or silent to decline sexual activity [36, 44]. Girls' culture of silence has been reported in Vietnam [57], and Ethiopia [44]. This silence has implications on girls' sexual health as it may hamper their rights related to SRHR [35], especially in reporting gender and sexual-based violence. The study also reported girls' weaknesses that call for protection from boys. This intergroup gender attitude has been documented in the gender development process where girls may be viewed as vulnerable and in need of protection on one side, and where boys want to be remarkable by their dominance and be admired as providers and heroes on the other side [58]. In addition, the findings depicted boys as socially allowed multiple sex partners. These findings corroborate those published by Khuzwayo and Taylor [59].

Imbalances also exist in gender roles that are different and specific for boys and girls. They are therefore socialized differently. Differences in expectations about boys' and girls' gender roles in Uganda, Ethiopia, Vietnam, Nepal, and India have been reported in previous research $[11,60]$. This is a traditional perspective of gender roles that support a division of family labour between men and women, boys and girls [61]. Such perspective reflects inequality between adolescent boys and girls, possible violence towards the partner in affective relationships, distrust in relationships, and justification of abusive behaviour [62]. Boys' domination and girls' submissive attitudes may confer boys a decision-making power on the types and frequency of sexual behaviours [42]. Furthermore, according to participants' perspectives in this study, girls experience cultural pressure for marriage. Studies have indicated an association between this pressure for early marriage and unequal power and gender norms [63]. Research in Uganda revealed that boys have also specific cultural pressures [60].

The study further indicated the lack of an agreed-upon definition of the concept of "gender" applicable to the Rwandan context. Clarifying this concept is crucial in setting appropriate curriculum gender content, and delivering standard gender knowledge. It is a foundation to anyone involved in gender education by determining what, how, when, and to whom, and who best to provide it [64].

This study revealed that early adolescents access multiple sources of SRHR information, including both informal and formal ones. This is consistent with research indicating the existence of numerous channels of SRHR information due to developments in science and technology, and globalization [65]. One important issue is related to the quality of SRHR information given to early adolescents by these sources. For example, school settings are considered as an official route for communication about sexuality, while information from peers is ambiguous and full of misperceptions [66]. The study found there were structured programs such as the school CSE initiated in 2016 [21], Youth Corner, Urunana, Ni Nyampinga, and Mobile for Reproductive Health $(\mathrm{m} 4 \mathrm{RH})$ that delivered reliable SRHR information to early adolescents. Ni Nyampinga, created by girls for girls, is a radio show that targets only girls by delivering information about health and relationships and depicts ideal girlhood for female early adolescents [67, 
68]. The $\mathrm{m} 4 \mathrm{RH}$ is a text message-based platform that involves sending $\mathrm{SRH}$ information to adolescents in the local language, Kinyarwanda [69]. However, the m4RH program has limitations related to low ownership of mobile phones among early adolescents. Despite the prominent role of parents in SRHR education $[11,51]$, this role was not significantly underscored by this study as sexuality is still taboo and inadequate communication of parents.

Similar to previous research $[52,70,71]$, this study also outlined the role of FBOs in shaping early adolescents' SRHR information in Rwanda, through focusing on moral values of abstinence, virginity, and rejecting the use of modern contraception. FBOs believe SRHR information to early adolescents encourages premarital sex $[39,43]$ while Government officials believe the information is beneficial in preventing unplanned pregnancy and STIs. Evidence suggests that religious beliefs may promote or inhibit the delivery of SRHR information $[72,73]$ and sexual behaviours $[41,74]$. This study found that FBOs enable selectively SRHR information delivery [43].

This study highlighted the importance of societal factors such as financial and human resources on SRHR education of early adolescents. The study noted challenges related to the development of books and shortages of employees to deliver SRHR information to early adolescents. In this regard, issues related to insufficient resources constitute challenges to the implementation of CSE in resource-poor settings [75-77]. Also, similar to other studies, countries implementing CSE had challenges in training teachers and supporting them with appropriate materials [78]. Finally, given the policy in the early adolescents' development and health [15], the study found that enabling SRHR education requires resolving policy issues of inadequate policy and laws contents, policy-setting processes through research, and by involving more stakeholders from the community to have a shared view of the ideal family. This would allow to development of a context-relevant CSE curriculum and accommodate external influences such as westernization [79]. Revising the laws would enable early adolescents to access services including SRHR information without parental consent as recommended by the World Health Organization [80]

\section{Conclusion}

In conclusion, the study revealed that most stakeholders had a favourable attitude towards early adolescents' access to SRHR information. Boys were reported to have more decision-making powers on sexual health. At the family level, girls were more prone to receiving gender and sexuality information than boys. Sources of SRHR information have diversified to deliver high-quality SRHR information to early adolescents. Religious institutions are still reluctant to afford to discuss some topics on sexuality with early adolescents. The role of parents is still minimal due to cultural restrictions. Access to SRHR information faces many challenges at the individual, family and peer, culture and religion, as well as the societal levels. More studies would ascertain the quality of SRHR information delivered and the extent to which each of these levels affects early adolescents' access to SRHR information. Such studies need to bring insights from different stakeholders such as healthcare providers, parents, school teachers, and early adolescents. 


\section{Abbreviations}

ADEPR: Association of Pentecostal Church of Rwanda

CSE: Comprehensive Sexuality Education

FBO: Faith-Based Organisations

ICPD: International Conference on Population and Development

m4RH: Mobile for Reproductive Health

NGOs: non-governmental organizations

RBC: Rwanda Biomedical Centre

SRHR: Sexual and Reproductive Health and Rights

\section{Declarations}

\section{Ethical approval and consent to participate}

This study obtained approvals from the Human Research Ethics Committee of the University of the Witwatersrand (study reference number: M190303) and the Institutional Review Board of the College of Medicine and Health Sciences of the University of Rwanda (approval notice number: 401/CMHS IRB/2018). The National Council for Science and Technology of Rwanda also provided permission to conduct this study (No. NCST/482/136//2019). All participants provided signed written informed consent forms before interviews. All methods in this study were performed in accordance with relevant ethical guidelines and regulations for research involving human subjects.

\section{Consent for publication}

Not applicable

\section{Availability of data and materials}

The dataset used and/or analysed during the current study are available from the corresponding author on reasonable request.

\section{Competing interests}

The authors declare that they have no competing interests. 


\section{Funding}

This research was supported by the Consortium for Advanced Research Training in Africa (CARTA). CARTA is jointly led by the African Population and Health Research Center and the University of the Witwatersrand and funded by the Carnegie Corporation of New York (Grant No--B 8606.R02), Sida (Grant No:54100113), the DELTAS Africa Initiative (Grant No: 107768/Z/15/Z) and Deutscher Akademischer Austauschdienst (DAAD). The DELTAS Africa Initiative is an independent funding scheme of the African Academy of Sciences (AAS)'s Alliance for Accelerating Excellence in Science in Africa (AESA) and supported by the New Partnership for Africa's Development Planning and Coordinating Agency (NEPAD Agency) with funding from the Wellcome Trust (UK) and the UK government.

\section{Authors' contributions}

VM, DNC and SG were involved in the conception and the design of the study. VM conducted interviews and drafted the initial version of the manuscript. DNC and SG provided support throughout the writing process of the manuscript, assisted in data analysis and interpretation. All authors read and approved the final manuscript.

\section{Acknowledgements}

We would like to acknowledge the support of public institutions, FBOs and one NGO in Rwanda for availing the study participants. We also Ms Aline Ikirezi for her great contribution in data transcription and translation.

\section{References}

1. Haberland, N. and D. Rogow, Sexuality Education: Emerging Trends in Evidence and Practice. Journal of Adolescent Health, 2015. 56(1, Supplement): p. S15-S21.

2. Ram, S., S. Andajani, and M. Mohammadnezhad, Parent's Perception regarding the Delivery of Sexual and Reproductive Health (SRH) Education in Secondary Schools in Fiji: A Qualitative Study. Journal of Environmental and Public Health, 2020. 2020: p. 1-8.

3. Breuner, C.C. and G. Mattson, Sexuality Education for Children and Adolescents. Pediatrics, 2016. 138(2): p. e20161348.

4. Kagesten, A., et al., Understanding Factors that Shape Gender Attitudes in Early Adolescence Globally: A Mixed-Methods Systematic Review. PLoS One, 2016. 11(6): p. e0157805.

5. Blum, R.W., et al., Achieving Gender Equality Requires Placing Adolescents at the Center. Journal of Adolescent Health, 2019. 64(6): p. 691-693.

6. Hong, E. and Y. Kang, Gender Differences in Sexual Behaviors in Korean Adolescents. Journal of Pediatric Nursing, 2017. 37: p. e16-e22. 
7. McCleary-Sills, J., et al., Gendered norms, sexual exploitation and adolescent pregnancy in rural Tanzania. Reproductive Health Matters, 2013. 21(41): p. 97-105.

8. Kaphagawani, N.C. and E. Kalipeni, Sociocultural factors contributing to teenage pregnancy in Zomba district, Malawi. Global Public Health, 2017. 12(6): p. 694-710.

9. De Meyer, S., et al., A cross-sectional study on attitudes toward gender equality, sexual behavior, positive sexual experiences, and communication about sex among sexually active and non-sexually active adolescents in Bolivia and Ecuador. Glob Health Action, 2014. 7: p. 24089.

10. Cottingham, J.C., Gender Aspects of Sexual and Reproductive Health, in International Encyclopedia of Public Health. 2017. p. 233-239.

11. Basu, S., et al., Learning to Be Gendered: Gender Socialization in Early Adolescence Among Urban Poor in Delhi, India, and Shanghai, China. Journal of Adolescent Health, 2017. 61(4, Supplement): p. S24-S29.

12. Thurston, M.D. and S. Allan, Sexuality and sexual experiences during gender transition: A thematic synthesis. Clinical Psychology Review, 2017.

13. Igras, S.M., et al., Investing in very young adolescents' sexual and reproductive health. Glob Public Health, 2014. 9(5): p. 555-69.

14. Palermo, T., et al., Examining determinants of gender attitudes: evidence among Tanzanian adolescents. BMC Women's Health, 2020. 20(1): p. 195.

15. Blum, R.W., et al., A conceptual framework for early adolescence: a platform for research. Int J Adolesc Med Health, 2014. 26(3): p. 321-31.

16. Rwanda Utilities Regulatory Authority (RURA), Annual Report 2017-2018. 2018, RURA: Kigali, Rwanda.

17. Republic of Rwanda Ministry of Education, National School Health Policy. 2014, Kigali: Ministry of Education. 44.

18. Republic of Rwanda Ministry of Health, National Reproductive Maternal, Newborn, Child and Adolescent Health (RMNCAH) Policy. 2018, Kigali: Ministry of Health. 73.

19. Republic of Rwanda Ministry of Gender and Family Promotion, National Gender Policy. 2010, Kigali: Ministry of Gender and Family Promotion.

20. Republic of Rwanda, The Constitution of the Republic of Rwanda of 2003 Revised in 2015. Official Gazette $n^{\circ}$ Special of 24/12/2015, 2015: p. 1-158.

21. Tabaro, C., The Rwandan Secondary School Competence-Based Curriculum: Knowledge, Skills and Attitudes to Incorporate in the University of Rwanda-College of Education Programs to Align them with the Current Curriculum. International Journal of Education and Practice, 2018. 6(2): p. 64-75.

22. Republic of Rwanda Ministry of Education, Competence-Based Curriculum Curriculum Framework Pre-primary to Upper Secondary. 2015, Kigali: Rwanda Education Board.

23. Pandey, P.L., H. Seale, and H. Razee, Exploring the factors impacting on access and acceptance of sexual and reproductive health services provided by adolescent-friendly health services in Nepal. 
PLOS ONE, 2019. 14(8): p. e0220855.

24. National Institute of Statistics of Rwanda (NISR) [Rwanda], Ministry of Health (MOH) [Rwanda], and ICF International, Rwanda Demographic and Health Survey 2014-15 Final Report. 2015, Rockville, Maryland, USA: NISR, MOH, and ICF International.

25. Pitotti, J.A., et al., Understanding Adolescent Sexual \& Reproductive Health Behavior \& Care Access in a Rwandan Community. Obstetrics and Gynecology, 2017. 129: p. 18S-19S.

26. De Meyer, S., et al., "Boys Should Have the Courage to Ask a Girl Out": Gender Norms in Early Adolescent Romantic Relationships. Journal of Adolescent Health, 2017. 61(4, Supplement): p. S42S47.

27. Mmari, K., et al., Exploration of Gender Norms and Socialization Among Early Adolescents: The Use of Qualitative Methods for the Global Early Adolescent Study. J Adolesc Health, 2017. 61(4S): p. S12S18.

28. Vu, L., et al., Inequitable Gender Norms From Early Adolescence to Young Adulthood in Uganda: Tool Validation and Differences Across Age Groups. Journal of Adolescent Health, 2017. 60(2, Supplement 2): p. S15-S21.

29. National Institute of Statistics of Rwanda (NISR) and Ministry of Finance and Economic Planning (MINECOFIN) [Rwanda], Rwanda Fourth Population and Housing Census. Thematic Report: Fertility. 2012, NISR: Kigali. p. 109.

30. National Institute of Statistics of Rwanda (NISR), EICV5 Main Indicators Report. 2018, NISR: Kigali. p. 190.

31. National Institute of Statistics of Rwanda (NISR) and Ministry of Finance and Economic Planning (MINECOFIN) [Rwanda], Rwanda Fourth Population and Housing Census. Thematic Report: Population size, structure and distribution. 2012, NISR \& MINECOFIN: Kigai. p. 102.

32. Republic of Rwanda, Law governing persons and family. Official Gazette n³7 of 12/09/2016, 2016.

33. Erlingsson, C. and P. Brysiewicz, A hands-on guide to doing content analysis. African Journal of Emergency Medicine, 2017. 7(3): p. 93-99.

34. Bengtsson, M., How to plan and perform a qualitative study using content analysis. NursingPlus Open, 2016. 2: p. 8-14.

35. Svanemyr, J., et al., Creating an enabling environment for adolescent sexual and reproductive health: a framework and promising approaches. J Adolesc Health, 2015. 56(1 Suppl): p. S7-14.

36. Khuzwayo, N. and M. Taylor, Exploring the socio-ecological levels for prevention of sexual risk behaviours of the youth in uMgungundlovu District Municipality, KwaZulu-Natal. African journal of primary health care \& family medicine, 2018. 10(1): p. e1-e8.

37. Markham, C.M., et al., Factors Associated With Early Sexual Experience Among American Indian and Alaska Native Youth. Journal of Adolescent Health, 2015. 57(3): p. 334-341.

38. Kemigisha, E., et al., Sexual health of very young adolescents in South Western Uganda: a crosssectional assessment of sexual knowledge and behavior. Reproductive Health, 2018. 15(1): p. 148. 
39. Bankole, A., et al., Sexual Behavior, Knowledge and Information Sources of Very Young Adolescents in Four Sub-Saharan African Countries. Afr J Reprod Health, 2007. 11(3): p. 28-43.

40. Yang, H.-t., et al., Status of reproductive health knowledge for junior high school students in Guangzhou city. Journal of Reproduction and Contraception, 2016. 27(1): p. 41-49.

41. Hong, J.S., et al., A Review of Sexual Attitudes, Knowledge, and Behaviors Among South Korean Early Adolescents: Application of the Ecological Framework. Journal of Social Service Research, 2016. 42(5): p. 584-597.

42. Lee, Y.-M., et al., Factors related to sexual behaviors and sexual education programs for AsianAmerican adolescents. Applied Nursing Research, 2015. 28(3): p. 222-228.

43. Motsomi, K., et al., Factors affecting effective communication about sexual and reproductive health issues between parents and adolescents in zandspruit informal settlement, Johannesburg, South Africa. Pan Afr Med J, 2016. 25: p. 120.

44. Kusheta, S., et al., Adolescent-parent communication on sexual and reproductive health issues and its factors among secondary and preparatory school students in Hadiya Zone, Southern Ethiopia: institution based cross sectional study. BMC Pediatr, 2019. 19(1): p. 9.

45. United Nations Educational Scientific and Cultural Organization, Emerging Evidence, Lessons and Practice in Comprehensive Sexuality Education A Global Review. 2015, Paris, France: UNESCO.

46. Bushaija, E., et al., Factors that Hinder Parents from the Communicating of Sexual Matters with Adolescents in Rwanda. Rwanda Journal of Health Sciences, 2013. 2(2): p. 13.

47. Bastien, S., L.J. Kajula, and W.W. Muhwezi, A review of studies of parent-child communication about sexuality and HIV/AIDS in sub-Saharan Africa. Reprod Health, 2011. 8: p. 25.

48. Ngilangwa, D.P., et al., Accessibility to sexual and reproductive health and rights education among marginalized youth in selected districts of Tanzania. Pan Afr Med J, 2016. 25(Suppl 2): p. 2.

49. Ashcraft, A.M. and P.J. Murray, Talking to Parents About Adolescent Sexuality. Pediatric clinics of North America, 2017. 64(2): p. 305-320.

50. Fonner, V.A., et al., School based sex education and HIV prevention in low- and middle-income countries: a systematic review and meta-analysis. 2014. 9(3): p. e89692.

51. Rusby, J.C., et al., Influence of parent-youth relationship, parental monitoring, and parent substance use on adolescent substance use onset. Journal of family psychology : JFP : journal of the Division of Family Psychology of the American Psychological Association (Division 43), 2018. 32(3): p. 310320.

52. Frederico, M., et al., Adult and young women communication on sexuality: a pilot intervention in Maputo-Mozambique. Reprod Health, 2019. 16(1): p. 144.

53. Widman, L., et al., Parent-Adolescent Sexual Communication and Adolescent Safer Sex Behavior: A Meta-Analysis. JAMA pediatrics, 2016. 170(1): p. 52-61.

54. Muhwezi, W.W., et al., Perceptions and experiences of adolescents, parents and school administrators regarding adolescent-parent communication on sexual and reproductive health issues 
in urban and rural Uganda. Reproductive Health, 2015. 12(1): p. 110.

55. Kajula, L.J., et al., Dynamics of parent-adolescent communication on sexual health and HIV/AIDS in Tanzania. AIDS Behav, 2014. 18 Suppl 1: p. S69-74.

56. Ministry of Health (MOH) [Rwanda], Rwanda Biomedical Center [RBC], and Imbuto Foundation, Tuganire Mwana Wanjye [Let's Talk, My Child]. 2016, Kigali: Imbuto Foundation. 44.

57. Bui, T.C., et al., Dimensions of gender relations and reproductive health inequity perceived by female undergraduate students in the Mekong Delta of Vietnam: a qualitative exploration. Int J Equity Health, 2012. 11: p. 63.

58. Martin, C.L. and D.N. Ruble, Patterns of gender development. Annual review of psychology, 2010. 61: p. 353-381.

59. Khuzwayo, N. and M. Taylor, Exploring the socio-ecological levels for prevention of sexual risk behaviours of the youth in uMgungundlovu District Municipality, KwaZulu-Natal. African Journal of Primary Health Care \& Family Medicine, 2018. 10(1): p. 1590.

60. Marcus, R., et al., Social norms, gender norms and adolescent girls: a brief guide. 2015, Overseas Development Institute: London.

61. Halimi, M., et al., The Relationship Between Youngsters' Gender Role Attitudes and Individual, Home, and School Characteristics:A Review. SAGE Open, 2016. 6(3): p. 2158244016656230.

62. García-Cueto, E., et al., Development of the Gender Role Attitudes Scale (GRAS) amongst young Spanish people. International Journal of Clinical and Health Psychology, 2015. 15(1): p. 61-68.

63. Ninsiima, A.B., et al., "Girls Have More Challenges; They Need to Be Locked Up": A Qualitative Study of Gender Norms and the Sexuality of Young Adolescents in Uganda. Int J Environ Res Public Health, 2018. 15(2).

64. Pop, M.V. and A.S. Rusu, The Role of Parents in Shaping and Improving the Sexual Health of Children - Lines of Developing Parental Sexuality Education Programmes. Procedia - Social and Behavioral Sciences, 2015. 209: p. 395-401.

65. Babayanzad Ahari, S., et al., Concerns and educational needs of Iranian parents regarding the sexual health of their male adolescents: a qualitative study. Reprod Health, 2020. 17(1): p. 24.

66. Warner, L.R., E.A. Leskinen, and J. Leyva, Sexuality and Socialization, in Companion to Sexuality Studies. 2020. p. 160-178.

67. McLean Hilker, L.C., Navigating adolescence and young adulthood in Rwanda during and after genocide: intersections of ethnicity, gender and age. Children's Geographies, 2014. 12(3): p. 354-368.

68. Boyd, G.G.-D., The Girl Effect: A Neoliberal Instrumentalization of Gender Equality. Consilience: The Journal of Sustainable Development, 2016. 15(1): p. 146-180.

69. L'Engle, K., K.F. Plourde, and T. Zan, Evidence-based adaptation and scale-up of a mobile phone health information service. Mhealth, 2017. 3: p. 11.

70. Rabbitte, M. and M. Enriquez, The Role of Policy on Sexual Health Education in Schools: Review. J Sch Nurs, 2019. 35(1): p. 27-38. 
71. Mturi, A.J. and A.L. Bechuke, Challenges of Including Sex Education in the Life Orientation Programme Offered by Schools: The Case of Mahikeng, North West Province, South Africa. 2019. 23(3): p. 134-148.

72. Zulu, J.M., et al., Why teach sexuality education in school? Teacher discretion in implementing comprehensive sexuality education in rural Zambia. International Journal for Equity in Health, 2019. 18(1): p. 116.

73. Ott, K. and D.W. Stephens, Embodied learning: Teaching sexuality and religion to a changing student body. Teaching Theology \& Religion, 2017. 20(2): p. 106-116.

74. Rahmani, A., E. Merghati-Khoei, and A. Fallahi, Perceived Advantages and Disadvantages of Sex Education in Young Women: A Qualitative Study. Int J High Risk Behav Addict, 2018. 7(2): p. e57221.

75. Keogh, S.C., et al., Challenges to implementing national comprehensive sexuality education curricula in low- and middle-income countries: Case studies of Ghana, Kenya, Peru and Guatemala. PloS one, 2018. 13(7): p. e0200513-e0200513.

76. Sidze, E.M., et al., From Paper to Practice: Sexuality Education Policies and Their Implementation in Kenya. 2017, Guttmacher Institute: New York.

77. Vanwesenbeeck, l., et al., Lessons learned from a decade implementing Comprehensive Sexuality Education in resource poor settings: The World Starts With Me. Sex Education, 2016. 16(5): p. 471486.

78. Keogh, S.C., et al., Measuring the quality of sexuality education implementation at the school level in low- and middle-income countries. Sex Education, 2020. 20(2): p. 119-137.

79. Fatusi, A.O. and M.J. Hindin, Adolescents and youth in developing countries: Health and development issues in context. J Adolesc, 2010. 33(4): p. 499-508.

80. WHO, WHO Recommendations on Adolescent Health. 2017, Geneva: World Health Organization. 